удК 330(075.8)

А. Е. Плахин

Уральский государственный эконолический университет, г. Екатеринбург, Российская Федерация

\title{
МЕТОДИЧЕСКИЕ ПОДХОДЫ К ВНЕДРЕНИЮ СИСТЕМ УПРАВЛЕНИЯ ИННОВАЦИЯМИ В ПРОЦЕССАХ РАЗВИТИЯ ПРОМЫШЛЕННЫХ КЛАСТЕРОВ
}

\begin{abstract}
АНнОТАЦИЯ. В статье обосновываются организационный и методологический подходы к созданию систем управления инновационным развитием промышленных кластеров, адаптивное применение которых позволит обеспечить достижение долгосрочных конкурентных преимуществ национальной экономики. Раскрывается содержание категории «управление инновационным развитием», указывается на важность и необходимость исследования проблем внедрения инноваций в процессы развития промышленных кластеров как социально-экономических систем, предполагающих обоснование создания комплекса механизмов, которые позволяют, с одной стороны, осуществлять имеющиеся научно-технические достижения на конкретном предприятии промышленного кластера, а с другой - формировать необходимую среду для генерации инновационных идей и активизации инвестиционной деятельности всего кластера. При этом с опорой на общие комбинации и принципы целостной системы национальной экономики основное внимание в исследовании уделяется не только внутрирегиональной специфике, учитываются также общемировые тенденции инновационного и инвестиционного развития промышленных кластеров.

кЛючЕВЫЕ СЛОВА. Инноватика; инвестиционный процесс; промышленные кластеры; инновационное развитие; инвестиционное развитие; методология; мониторинг; организация; программно-целевой подход; социально-экономическая система; управление; кластеры.

ФИНАНСИРОВАНИЕ. Статья подготовлена при поддержке РГНФ в рамках гранта «Разработка стратегии взаимодействия промышленных кластерных формирований Свердловской области и Республики Беларусь в рамках Союзного государства» (проект № 16-07-00018).

ИНФОРМАЦИЯ О СТАТЬЕ. Дата поступления 2 марта 2017 г.; дата принятия к печати 17 марта 2017 г.; дата онлайн-размещения 31 марта 2017 г.
\end{abstract}

A. E. Plakhin

Ural State University of Economics, Yekaterinburg, Russian Federation

\section{METHODOLOGICAL APPROACHES TO IMPLEMENTING INNOVATION MANAGEMENT SYSTEMS IN PROCESSES OF INDUSTRIAL CLUSTERS DEVELOPMENT}

\begin{abstract}
The article substantiates organizational and methodological approaches to building up management systems for innovative development of industrial clusters, adaptive use of which will allow to achieve long-term competitive advantage of the national economy. It reveals the content of the category «management of innovative development», points out to importance and necessity of studying the problems of implementing innovations in the processes of industrial clusters development as socio-economic systems that assume the substantiation of building up a complex of mechanisms that allow, on the one hand, to implement the available scientific and technological achievements in a particular enterprise of the industrial cluster, and on the other hand, to form the necessary environment for generating innovative ideas and activating investment activities of the entire cluster. At the same time, when supported by general combinations and principles of the integrated system of the national economy the re-
\end{abstract}

(C) A. Е. Плахин, 2017

\section{Baikal Research Journal}

электронный научный журнал Байкальского государственного университета 
search places emphasis not only on the intraregional specifics, it also takes into account the global trends in innovation and investment development of industrial clusters.

KEYWORDS. Innovation; the investment process; industrial clusters; innovative development; investment development; methodology; monitoring; organization; target-oriented approach; socio-economic system; management; clusters.

FINANCING. This paper has been supported by the Russian Humanitarian Science Foundation, «Strategy development for collaboration between the industrial cluster entities of Sverdlovsk region and the Republic of Belarus within the framework of the Union State» (Project no. 16-07-00018).

ARTICLE INFO. Received March 2, 2017; accepted March 17, 2017; available online March 31, 2017.

Форсирование научно-технического прогресса, нестабильность мировой экономики, возрастание рыночной конкуренции и другие значимые изменения создают условия для формирования новых подходов к механизмам образования и реализации бизнес-процессов и существенно преобразуют привычные формы хозяйственных отношений, а также меняют стиль и образ жизни современного общества.

Активное использование инноваций, генерирование новых знаний, формирование интеллектуального капитала и последующая его трансформация в результативное производство являются предпосылками для становления инновационного технического уклада, способного в дальнейшем обеспечить привилегированность конкурентных преимуществ национальной экономики и ее переход на инновационный путь развития.

В сложившихся условиях предметом пристального интереса ученых, представителей органов государственной власти и бизнес-структур являются вопросы, касающиеся проблем реализации интенсивного типа экономического роста и усовершенствования качества жизни народонаселения в постиндустриальной экономике. Соответственно, актуальность разработки научно доказанной методологии управления инновационным развитием промышленных кластеров как социально-экономических систем становится очевидной ${ }^{1}[1-6]$. Она позволит обеспечить их дальнейшее устойчивое функционирование в условиях неопределенности факторов внешней и внутренней среды, а также будет способствовать необходимому приращению знаний в современной теории управления и экономической науке в целом.

На текущий момент представлено значительное количество определений понятия «управление», в контексте которых оно является элементом, деятельностью, функцией, процессом, противодействием, выбором, результатом и т. д., т. е. изучается в качестве науки, практики или конкретного объекта. В целях настоящего исследования понятие «управление» рассматривается как целенаправленное действие субъекта управления (управляющей системы) на объект управления (управляемую систему) для решения проблем инновационного развития. Использование разных подходов к управлению предусматривает учет определенных закономерностей развития социально-экономических систем как источника инноваций [7]:

- неравномерность развития разных функциональных свойств системы и направлений деятельности;

- гетерохронность как асинхронность фаз развития единичных структурных элементов системы и ее функциональных направлений;

- волнообразный тип развития системы в условиях риска и неопределенности, включая кризисные явления;

- восприимчивость, высокая степень чувствительности объекта управления к внешним воздействиям;

1 The Global Startup Ecosystem Ranking 2015. URL: http://www.rusventure.ru/ru/programm/ analytics/docs/Report_RVC_2014.pdf.

\section{Baikal Research Journal}

электронный научный журнал Байкальского государственного университета 
- кумулятивность, определяемая потенциалом, накопленным в предшествующие фразы развития;

- дивергенция (диверсификация), предполагающая увеличение разнообразия инноваций в процессе прогрессирования системы;

- конвергенция (разделение) - увеличение избирательности инновационной деятельности и др.

Производственные, логистические, энергетические, финансово-инвестиционные, инфраструктурные и другие системы чаще всего выступают в качестве приоритетных объектов управления инновационным развитием. Данный факт позволяет решать многоаспектные задачи инновационной деятельности промышленного кластера как объекта исследования.

В рамках изучаемого направления объектом управления инновационным развитием становятся промышленные кластеры с учетом особенностей их внутренней структуры и взаимосвязей с внешней средой.

Объект управления инновационным развитием в качестве открытой системы, взаимодействующей с окружающей средой, находится в непрерывном развитии:

- меняется динамика жизнедеятельности;

- усложняются связи и структура;

- возникают новые свойства и процессы.

Инновационное развитие промышленных кластеров можно охарактеризовать как необратимость, нелинейность и специфичность. Образование у них новых качеств в ходе развития фактически означает переход из одного состояния в другое, сопровождающееся изменением состава или структуры. При этом главным свойством инновационного развития кластеров является их изменение во времени, поскольку, во-первых, всякое развитие реализуется в реальном времени и, во-вторых, именно время во многом определяет направление развития [8-12].

Следовательно, организация управления должна рассматриваться как комплекс организационно-распорядительных функций, позволяющих систематизировать процесс управления и соединять в единое целое все его элементы на всех уровнях, обеспечивая при этом эффективность управления, и динамичное инновационное развитие промышленного кластера в целом. В свою очередь, управление объектом в контексте инновационного развития представляет собой единство процессов управленческого цикла и форм его организации, направленное на разработку и внедрение нововведений за счет комплексного воздействия на составляющие элементы кластера с учетом содержательно-смыслового и динамического компонентов.

Изложенное позволяет перейти к формированию методологических аспектов исследования внедрения инноваций в процесс развития промышленных кластеров.

Проведенные исследования прототипа механизма осуществления инновационно-инвестиционных проектов среди инструментов управления социально-экономическим развитием (прогноз - концепция - план - программа) указывают на необходимость проработки вопросов, связанных с функционированием ее завершающего элемента - программного комплекса, «вписанного» в общую модель государственного управления экономикой [13-16].

Считаем целесообразным осуществлять решение данной задачи на основе так называемого программно-целевого подхода с учетом специфики рассматриваемой проблемы. Принципиальным моментом при этом должна стать идентификация «целевой траектории», которая определяет общую «идеологию» создания программ целевого развития.

Представляется, что формирование траектории реализации программно-целевого подхода должно происходить в следующей последовательности:

1. Постановка проблемы.

\section{Baikal Research Journal}

электронный научный журнал Байкальского государственного университета 
2. Исследование особенностей.

3. Разработка комплекса задач.

4. Создание конструктивной модели процесса развития промышленного кластера.

5. Составление алгоритма процесса развития и выбор методов решения поставленных задач.

6. Формулировка программных мероприятий.

Детализация каждого из звеньев в совокупности позволяет сформировать основу рекомендуемой методики разработки программ целевого развития (ПЦР) промышленного кластера. Исходной точкой должно стать установление перечня проблем, подлежащих решению при создании и практическом осуществлении ПЦР кластера. При проведении исследования было выделено 7 основных проблем и проведена их систематизация (рис. 1). Подходы к решению некоторых из них (например - 1, 2 и 3) на концептуальном уровне могут быть использованы при разработке конкретных ПЦР. В то же время другие проблемы (например $-4,5$, 6 и 7) требуют «приближения» к специфике среды их реализации и учета особенностей функционирования кластера при внедрении инноваций. В связи с этим необходима декомпозиция во второй точке обозначенной траектории до общепроблемного уровня, что позволит перейти к формулировке задач, подлежащих решению при построении механизма управления реализацией инноваций.

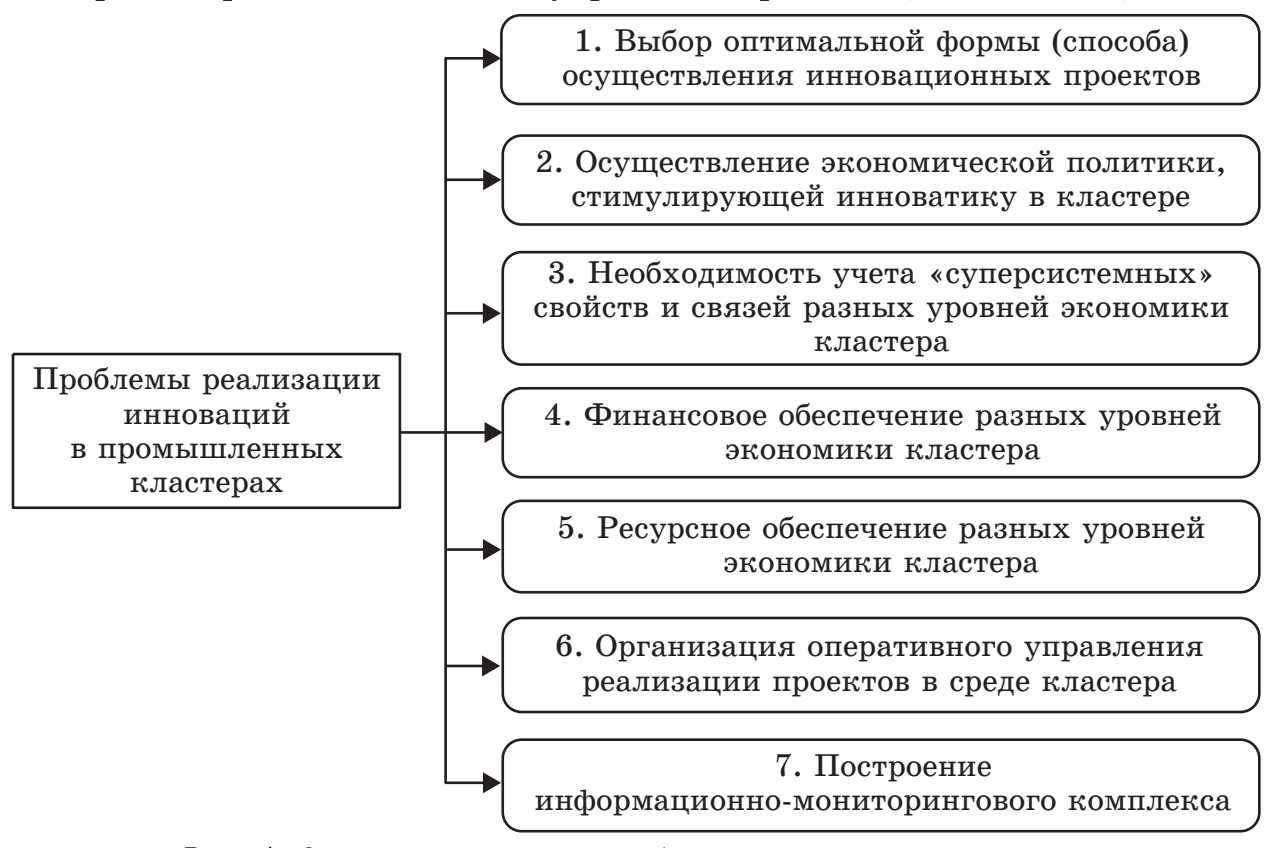

Рис. 1. Систелатизация проблем реализации инноваций в промышленном кластере

C учетом сказанного можно представить структурную модель, отражающую взаимосвязь выявленных особенностей, системно проявляющихся при решении обозначенных проблем на всех уровнях социально-экономической системы (рис. 2).

Данная пятикомпонентная структура является универсальной и предполагает возможность дальнейшей декомпозиции до более детальных элементов исходя из условий и целей конкретной ПЦР.

\section{Baikal Research Journal}

электронный научный журнал Байкальского государственного университета 


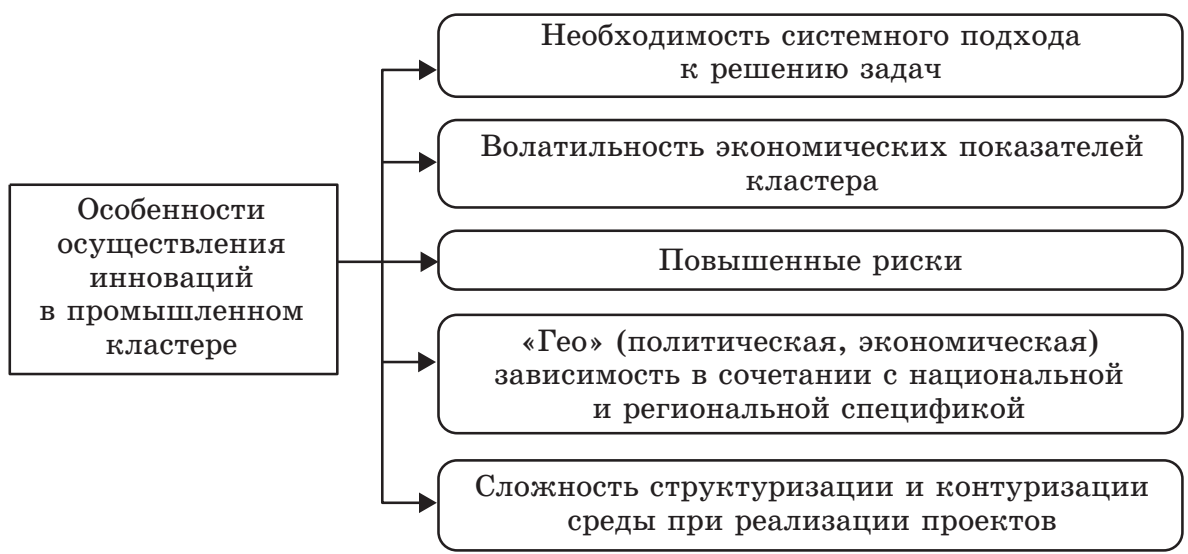

Рис. 2. Структурная модель взаимосвязи особенностей осуществления инноваций в промышленнол кластере

Следующей точкой в рекомендуемой траектории реализации программно-целевого подхода является определение и формулировка основных задач, решение которых будет обеспечивать возможность создания модели «управляемой системы инновационно-инвестиционного развития», которая станет базой для разработки соответствующих программных мероприятий.

На основе проведенных исследований [17-21] определен комплекс задач, требующих решения для построения модели механизма управления реализацией инноваций в соответствующей среде промышленного кластера. Данный комплекс состоит из 6 позиций (рис. 3) и основывается на модели, описывающей структуру и взаимодействие системных компонентов среды реализации инвестиционных проектов.

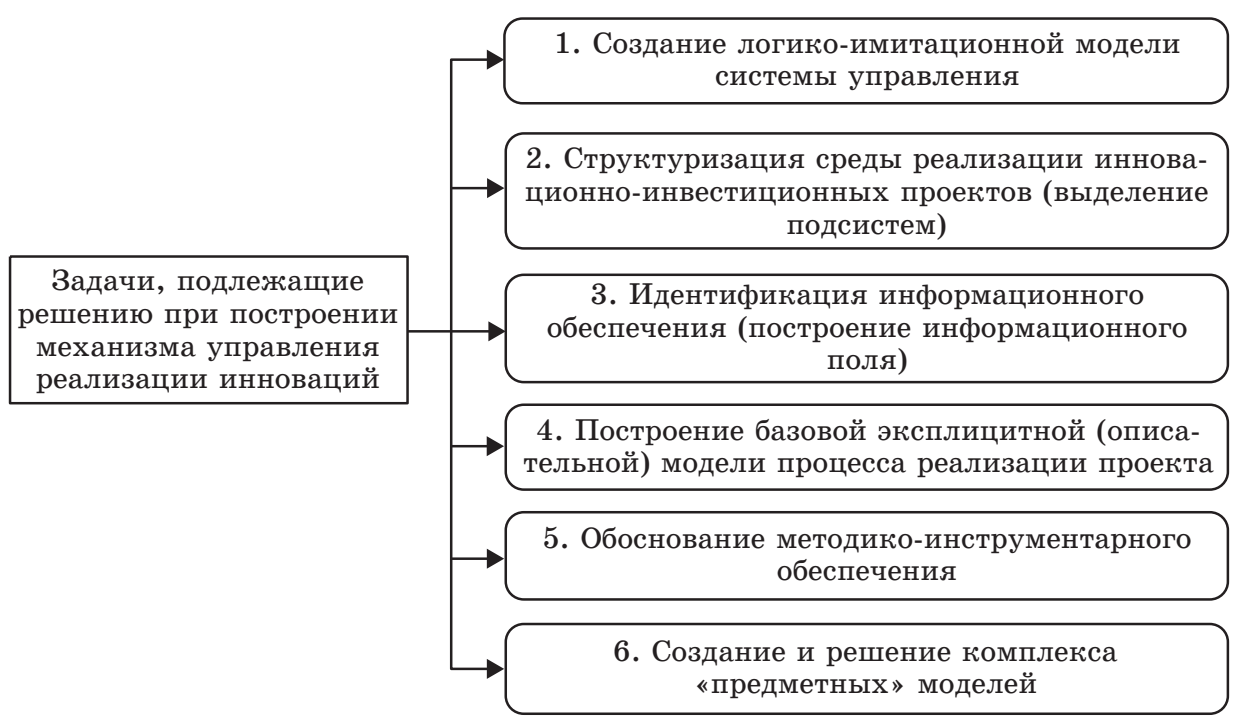

Рис. 3. Комплекс задач, требующих решения для построения модели механизма управления реализацией инноваций в промышленном кластере

В данном комплексе важным является решение первых четырех задач, приводящее в итоге к конструированию базовой модели, декомпозированной до уровней, необходимых для понимания, с позиций управления происходящими про-

\section{Baikal Research Journal}


цессами. В связи с этим представляется целесообразным провести детализацию «методологической» модели структуризации среды реализации инвестиционных проектов, состоящей из двух подсистем - квалификационной и ресурсной, путем выделения третьей - операционной подсистемы и информационных полей с учетом результатов проведенных исследований.

Преимуществом предлагаемого подхода является сформированная конструкция «рабочей» эксплицитной модели, в которой показано функционирование операционной подсистемы при продвижении проекта по этапам жизненного цикла и построение информационных полей в увязке с выделенными уровнями среды промышленного кластера (CP1-CP4) и блоками квалификационной и ресурсной подсистем (рис. 4).

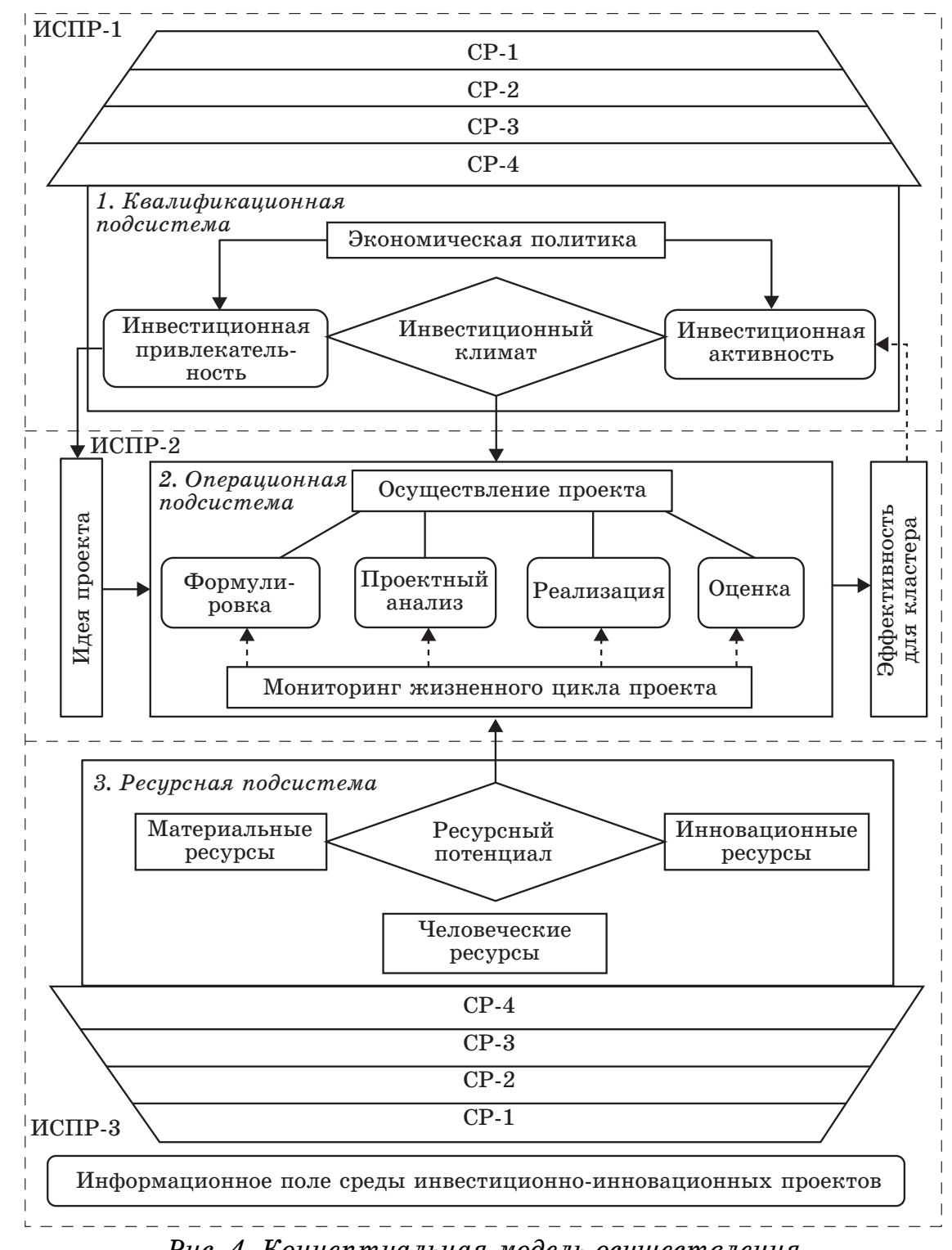

Рис. 4. Концептуальная модель осуществления

инновационно-инвестиционных проектов в прольшленнол кластере

\section{Baikal Research Journal}


«Пирамидальное» конструирование информационных полей для квалификационной (ИПСР-1) и ресурсной (ИПСР-2) подсистем обусловлено разной степенью детализации и количеством показателей, соответственно, в страновом (CP-1), peгиональном (CP-2), субъектном (CP-3) и муниципальном (CP-4) уровнях системы.

Предлагаемая разработка представляет собой суть четвертой точки траектории реализации программно-целевого подхода и позволяет перейти к этапам алгоритмизации и формализации решений. На основе этой модели с учетом решений в точках 1,2 и 3 целевой траектории формулируется алгоритм «предметного моделирования» для разработки конкретных решений по механизмам мониторинга, управления и развития (точка 5 целевой траектории).

Укрупненно алгоритм образует следующую последовательность:

- разработка системной модели осуществления инновационно-инвестиционного проекта;

- структуризация выделенной системы;

- информационно-аналитическая контуризация для построения «управляемой» системы;

- формирование комплекса показателей для идентификации информационных полей среды реализации (ИПСР-1, ИПРС-2, ИПСР-3);

- разработка рекомендаций по формированию программных мероприятий, механизмов мониторинга и управления.

Следует подчеркнуть, что инновационно-инвестиционная деятельность, направленная на обеспечение экономической и социальной выгоды, для промышленного кластера играет ведущую роль в устойчивом развитии экономики региона. Инновационно-инвестиционный процесс в силу своей природы ориентирован на длительную временную перспективу, которую невозможно рассматривать в отрыве от глобальных трансформационных процессов.

Выводы и перспективы дальнейших исследований. Необходимо прогнозирование инновационной деятельности промышленных кластеров с учетом влияния на нее различных внутренних и внешних факторов. Требуется постоянно искать источники инвестирования, которые должны быть направлены на конкретные инновации и нововведения. Развитие инновационно-инвестиционных процессов возможно только в кластерах, обладающих большим производственным и интеллектуальным потенциалом.

В свою очередь развитие кластеров в значительный степени зависит от инструментов государственного воздействия на создание соответствующего экономического механизма. Большое значение в процессе воспроизводства интеллектуального капитала имеет научно-техническая политика, проводимая как на уровне государства, так и на уровне регионов.

Рассматриваемая проблема совершенствования методологии управления развитием промышленных кластеров как источников инноваций важна, поскольку ее решение положительно скажется на обеспечении эффективного функционирования всей национальной экономики в нестабильной рыночной среде, а разработанная методика построения моделей систем управления для создания механизма инновационно-инвестиционного развития позволит более обстоятельно взглянуть на возможности применения программно-целевого подхода в увязке с динамикой интеграционных процессов в экономике.

\section{Список используемой литературы}

1. Кластерная политика как метод активизации инновационных процессов / М. Алибаева, А. Алибаева, Б. Дусматов, Д. Одинаев // Вестник Технологического университета Таджикистана. - 2010. - № 3. - С. 96-102.

\section{Baikal Research Journal}

электронный научный журнал Байкальского государственного университета 
2. Баранов В. В. Формирование стратегии инновационной деятельности предприятий в различных отраслях российской экономики / В. В. Баранов, С. Н. Соколов, Д. А. Антипов // European Social Science Journal. - 2012. - № 5 (май). - C. 387-392.

3. Токтамышева Ю. С. Оценка реализации ориентированного развития экономики в регионах России / Ю. С. Токтамышева // Актуальные проблемы экономики и права. 2015. - № 2. - C. 118-125.

4. Rosenfeld S. A. Bringing business clusters into the mainstream of economic development / S. A. Rosenfeld // European Planning Studies. - 1997. - Vol. 5, № 1. - P. 3-23.

5. Sri Herliana. Regional innovation cluster for small and medium enterprises (SME): a triple helix concept / Sri Herliana // Procedia - Social and Behavioral Sciences. - Elsevier Ltd., 2015. - Vol. 169. - P. 151-160.

6. Xu Youli. Research on evaluation of enterprises' technology innovation performance from the perspective of industrial cluster networks / Xu Youli, Li Huiwei // Energy Procedia. - 2011. - Vol. 5. - P. 1279-1283.

7. Жура С. Е. Как оценить управление социально-экономическими системами? / С. Е. Жура // Российское предпринимательство. — 2010. — № 12-2. — С. 11-16.

8. Даваасурэн А. Развитие регионов путем создания инновационных промышленных кластеров / А. Даваасурэн // Вестник Бурятского государственного университета. 2014. - № 4-1. - С. 12-15.

9. Никулина О. В. Управление инновационным развитием промышленных предприятий в условиях формирования инновационного кластера в регионе / О. В. Никулина, К. И. Шевченко // Национальные интересы: приоритеты и безопасность. - 2010. № 28. - C. $38-49$.

10. Смирнов Е. Н. Особенности формирования региональной инновационной политики в условиях глобализации / Е. Н. Смирнов // Журнал правовых и экономических исследований. - 2013. - № 3. - С. 145-146.

11. Фонотов А. Г. Роль государственной научно-технической политики в повышении инновационной активности российских предприятий / А. Г. Фонотов // Проблемы прогнозирования. - 2013. - № 3. - С. 35-47.

12. Церцеил Ю. С. Роль инновационных промышленных кластеров в развитии региональной экономики / Ю. С. Церцеил, В. В. Коокуева // Российское предпринимательство. - 2016. - Т. 17, № 20. — С. 2779-2796.

13. Засимкина А. С. Государственное управление инновационно-инвестиционными проектами / А. С. Засимкина // Экономика. Бизнес. Банки. - 2016. - Т. 11. - С. 60-67.

14. Малинина С. Е. Категория инновационно-инвестиционных проектов в оценке эффективности / С. Е. Малинина // Actual Science. - 2016. - T. 2, № 12. - С. 251-252.

15. Поташник Я. С. Отбор инновационно-инвестиционных проектов с учетом их влияния на прибыльность предприятия в постпроектном периоде / Я. С. Поташник, Г. С. Храбан // Вестник Мининского университета. - 2016. - № 1-1 (13). - С. 12.

16. Фраймович Д. Ю. Оценка роли различных инвестиционных процессов в инновационном развитии российских регионов / Д. Ю. Фраймович // Социальные и гуманитарные знания. - 2016. - Т. 2, № 1 (5). - С. 13-19.

17. Басовский Л. Е. Прогнозирование и планирование в условиях рынка : учеб. пособие / Л. Е. Басовский. - М. : Инфра-М, 2014. - 260 с.

18. Гладилин А. В. Методические подходы к внедрению инноваций в процессы развития социально-экономических систем / А. В. Гладилин, О. Н. Коломыц // Экономика и предпринимательство. - 2015. - № 6 (59-1). — С. 96-99.

19. Миронов Д. С. Государственно-частное партнерство как элемент механизма формирования и развития технопарковых структур / Д. С. Миронов // Успехи современной науки. - 2016. - Т. 2, № 4. - С. 49-56.

20. Огородникова Е. С. Инфраструктурное обеспечение процессов модернизации региональной экономики : монография / Е. С. Огородникова, М. М. Сидоренко. - Екатеринбург : Изд-во Урал. гос. экон. ун-та, 2013. - 169 с.

21. Плахин А. Е. Инновационная активность крупного бизнеса в России / А. Е. Плахин, Е. Ф. Маджар // Инновации в создании и управлении бизнесом : материалы 3-й Междунар. науч. конф. преподавателей, сотрудников и аспирантов. - М. : Рос. ун-т дружбы народов (РУДН), 2012. - С. 156-163.

\section{Baikal Research Journal}




\section{References}

1. Alibayeva M., Alibayeva A., Dusmatov B., Odinayev D. Cluster policy as a method of activating innovative processes. Vestnik Tekhnologicheskogo universiteta Tadzhikistana= Bulletin of Tajikistan Technological University, 2010, no. 3, pp. 96-102. (In Russian).

2. Baranov V. V., Sokolov S. N., Antipov D. A. Strategy development for innovative activity of businesses in various sectors of Russian economy. European Social Science Journal, 2012, no. 5, pp. 387-392. (In Russian).

3. Toktamysheva Yu. S. Evaluation of implementing the strategy of economic innovationoriented development in Russian regions. Aktual'niye problemy ekonomiki $i$ prava $=$ Topical Issues of Economics and Law, 2015, no. 2, pp. 118-125. (In Russian).

4. Rosenfeld S. A. Bringing business clusters into the mainstream of economic development. European Planning Studies, 1997, vol. 5, no. 1, pp. 3-23.

5. Sri Herliana. Regional innovation cluster for small and medium-sized enterprises (SME): a triple helix concept. Procedia - Social and Behavioral Sciences. Elsevier Ltd., 2015, vol. 169, pp. 151-160.

6. $\mathrm{Xu}$ Youli, Li Huiwei. Research on evaluation of enterprises' technology innovation performance from the perspective of industrial cluster networks. Energy Procedia, 2011, vol. 5, pp. 1279-1283.

7. Zhura S. Ye. The problems of assessing effectiveness of social and economic systems management? Rossiiskoe predprinimatel'stvo = Russian Entrepreneurship, 2010, no. 12-2, pp. 11-16. (In Russian).

8. Davaasuren A. Regional development through establishing innovative industrial clusters. Vestnik Buryatskogo gosudarstvennogo universiteta $=$ Bulletin of Bryansk State University, 2014, no. 4-1, pp. 12-15. (In Russian).

9. Nikulina O. V., Shevchenko K. I. Managing innovative development of industrial businesses in terms of innovative cluster formation in the region. Natsional'nye interesy: prioritety i bezopasnost' = National Interests: Priorities and Security, 2010, no. 28, pp. 38-49. (In Russian).

10. Smirnov E. N. Specific features of forming regional innovation policy in terms of globalization. Zhurnal pravovykh $i$ ekonomicheskikh issledovanii = Journal of Legal and Economic Studies, 2013, no. 3, pp. 145-146. (In Russian).

11. Fonotov A. G. Role of state scientific and technological policy in improving innovation activity of Russian enterprises. Problemy prognozirovaniya = Studies on Russian Economic Development, 2013, no. 3, pp. 35-47. (In Russian).

12. Tsertseil Yu. S., Kookuyeva V. V. Role of innovative industrial clusters for development of regional economy. Rossiiskoe predprinimatel'stvo = Russian Entrepreneurship, 2016, vol. 17, no. 20, pp. 2779-2796. (In Russian).

13. Zasimkina A. S. Government management of innovation and investment projects. Ekonomika. Biznes. Banki = Economy. Business. Banks, 2016, vol. 11, pp. 60-67. (In Russian).

14. Malinina S. E. Category of innovative investment projects in efficiency evaluation. Actual Science, 2016, vol. 2, no. 12, pp. 251-252. (In Russian).

15. Potashnik Ya. S., Khraban G. S. Selecting innovation and investment projects in view of their impact on profitability of the enterprise in the post-project period. Vestnik Mininskogo universiteta $=$ Bulletin of Minin University, 2016, no. 1-1 (13), p. 12. (In Russian).

16. Fraimovich D. Yu. Assessing role of various investment processes in innovative development of Russia's regions. Sotsial'nye i gumanitarnye znaniya = Social and Humanitarian Knowledge, 2016, vol. 2, no. 1 (5), pp. 13-19. (In Russian).

17. Basovsky L. E. Prognozirovanie i planirovanie $v$ usloviyakh rynka [Forecasting and planning in market environment]. Moscow, Infra-M Publ., 2014. 260 p.

18. Gladilin A. V., Kolomyts O. N. Methodological approaches to innovations in development processes of socio-economic systems. Ekonomika $i$ predprinimatel'stvo = Economics and Entrepreneurship, 2015, no. 6 (59-1), pp. 96-99. (In Russian).

19. Mironov D. S. Public-private partnership as an element of the mechanism for building up and developing techno park structures. Uspekhi sovremennoi nauki = Modern Science Success, 2016, vol. 2, no. 4, pp. 49-56. (In Russian).

\section{Baikal Research Journal}

электронный научный журнал Байкальского государственного университета 
20. Ogorodnikova E. S., Sidorenko M. M. Infrastrukturnoe obespechenie protsessov modernizatsii regional'noi ekonomiki [Infrastructural provision of modernizing regional economy]. Yekaterinburg, Ural State University of Economics Publ., 2013. 169 p.

21. Plakhin A. E., Madzhar E. F. Innovative activity of big business in Russia. Innovatsionnaya aktivnost' krupnogo biznesa $v$ Rossii. Innovatsii $v$ sozdanii i upravlenii biznesom. Materialy 3-i Mezhdunarodnoi nauchnoi konferentsii prepodavatelei, sotrudnikov $i$ aspirantov [Innovations in business creation and management. Materials of the 3rd International Research Conference of Faculty members, Staffers and PhD Students]. Moscow, People's Friendship University of Russia Publ., 2012, pp. 156-163. (In Russian).

\section{Информация об авторе}

Плахин Андрей Евгеньевич - кандидат экономических наук, доцент, кафедра менеджмента, заместитель директора института менеджмента и информационных технологий, Уральский государственный экономический университет, 620144, г. Екатеринбург, ул. 8 Марта, 62, e-mail: apla@usue.ru.

\section{Author}

Andrey E. Plakhin - PhD in Economics, Associate Professor, Chair of Management, Deputy Director of Institute of Management and Information Technology, Ural State University of Economics, 62 8-th March St., 620144, Yekaterinburg, Russian Federation; e-mail: apla@usue.ru.

\section{Библиографическое описание статьи}

Плахин А. Е. Методические подходы к внедрению систем управления инновациями в процессах развития промышленных кластеров / A. Е. Плахин // Baikal Research Journal. - 2017. - T. 8, № 1. - DOI: 10.17150/2411-6262.2017.8(1).13.

\section{Reference to article}

Plakhin A. E. Methodological approaches to implementing innovation management systems in processes of industrial clusters development. Baikal Research Journal, 2017, vol. 8, no. 1. DOI: 10.17150/2411-6262.2017.8(1).13. (In Russian).

\section{Baikal Research Journal}

